\title{
An Image Dehazing Method Based on Atmospheric Veil Shifeng $\mathrm{Li}^{1, \mathrm{a}^{*}}$, Dengyin Zhang ${ }^{1, \mathrm{~b}}$, Mingye $\mathrm{Ju}^{1, \mathrm{c}}$ \\ ${ }^{1}$ Nanjing University of Posts and Telecommunications, Nanjing 210003, China a375010173@qq.com, bzhangdy@njupt.edu.cn, ${ }^{c} 1493012608 @ q q . c o m$
}

\section{Keywords: Image dehazing, Total variation model, Atmospheric veil, Haze density feature}

Abstract. At present, most of the image dehazing treatments have residual haze at depth of field and obvious over-enhancement phenomenon in bright area. To solve these problems, we propose a new method of fast image dehazing based on the atmospheric veil. Firstly, we use the total variation model to estimate the atmosphere veil, which can preserve the edge characteristics of image on the premise of improving the computational efficiency. Then, aiming at the failure of bright area of the atmosphere veil, we propose a fault-tolerant mechanism based on haze density feature to improve the universality of the algorithm. The experimental results show that our method has better visual effect than some existing mainstream dehazing techniques, and its efficiency also has obvious advantages.

\section{Introduction}

In recent years, a single image dehazing technique based on the atmospheric scattering model has made great progress. He[1] first proposed the basic concept based on the dark channel prior after a large number of statistical tests and it is possible to perform a good recovery effect with this prior knowledge. However, the soft-matting algorithm involved in this algorithm has very high space-time complexity, which makes the algorithm not real-time. To solve this problem, He[2] used a filter with edge-oriented function to replace the original algorithm in the matting process, although reducing the computational complexity, but at the expense of degrading the visual effect. Tarel[]ㅡ used the median filter to estimate the atmospheric veil, but it shows poor edge preserving performance, and improper parameter setting can easily produce the Halo effect. $\mathrm{Ju}[4]$ improved the scattering model and proposed a new image dehazing algorithm. Although the algorithm solves the problem of nonuniform illumination, it also introduces over-exposure phenomenon. So it is not stable.

In this paper, we propose a new method to achieve fast haze removal based on Tarel's algorithm that still has residual haze at depth of field and the over-enhancement phenomenon in the bright area. The experimental results show that our method can effectively preserve the edge characteristics of the restored image. It has better visual effect and lower computational complexity.

\section{Background}

\section{Atmospheric Scattering Model}

In machine vision and graphics, the atmospheric scattering model is widely used in image dehazing technology research, and its imaging equation can be described as[5]:

$$
I(x)=J(x) t(x)+A(1-t(x)) .
$$

where $I(x)$ is the hazy image, $A$ is the global atmospheric light, $J(x)$ is the hazefree image and $t(x)$ is the transmission map. When the atmosphere is homogeneous and uniform, $t(x)$ can be expressed as: $t(x)=e^{-k d(x)}$. where $d(x)$ is the depth of the scene, $k$ is the scattering coefficient of the medium.

In Equation (1), the atmospheric scattering model is composed of two parts: The first term of the right $J(x) t(x)$ is called direct attenuation, which indicates the scene reflected light that is not scattered. It is exponential decay with the increase of the depth of the scene. The second term $A(1-(x))$, 
known is as the atmospheric veil, indicates that the ambient light participates in the imaging part. It can degrade image contrast and blur the scene.

\section{The basic principles and defect analysis of Tarel's algorithm}

Based on the atmospheric scattering model, the image dehazing aims to recover the clear image $J(x)$ from the hazy image. From Equation(1), we only know $I(x)$. In order to recover $J(x)$, it is necessary to estimate $A$ and $t(x)$. But $t(x)$ is related to the depth of the scene and the scattering coefficient of the medium and can not be directly estimated. To solve this ill-posed problem, Tarel introduced the atmospheric veil $V(x)$. Therefore, $t(x)$ can be indirectly obtained by estimating $V(x)$ and $A \cdot V(x)$ is described as: $V(x)=A(1-t(x))$. The restored image after dehazing is better to preserve a certain amount of haze to make it look more real by introducing a adjustment factor $\omega(0<\omega<1)$. Then, $t(x)$ can be written as:

$$
t(x)=1-\omega \frac{V(x)}{A} .
$$

For $\mathrm{t}(\mathrm{x})$ contains too much texture noise, Tarel used a median filter with linear complexity to smooth operation. But the median filter does not have the edge preserving properties, which is very easy to make the restored image residual haze in depth discontinuity.

In addition, the existing dehazing methods set the adjustment factor $\omega$ to a fixed value (as in the He's method, $\omega$ is 0.95 ). But experiments show that the selection of different values of $\omega$ can make the subjective visual effect of restored image greatly different. This means that the fixed value of $\omega$ can not meet all scenarios, which should be treated as different values depending on the situation.

\section{The proposed approach}

\section{The estimate of the atmospheric veil}

It is easy to know from the literature[3] that the atmospheric veil is affected by two constraints: 1)for each pixel, $\mathrm{V}(\mathrm{x})$ is positive $(\mathrm{V}(\mathrm{x}) \geq 0) ; 2) \mathrm{V}(\mathrm{x})$ is not greater than the minimum color component $\mathrm{W}(\mathrm{x})$ of the hazy image. We make the initial estimate of $\mathrm{V}$ as:

$$
\mathrm{V}(\mathrm{x})=W(x)=\min _{C \in\{R, G, B\}}\left(I^{C}(x)\right)
$$

where $I^{c}(x)$ is the pixel of the channel $C$ at point $x$ of image $I$. As pointed out in Section 2, it is necessary to carry out edge-preserving smoothing operation on $\mathrm{V}$, which is to protect the edge information, as far as possible to eliminate the texture noise. Considering that the total variation model[4] just has this function, we define the energy function as:

$$
E(\tilde{V})=\frac{\alpha}{2}\|\tilde{V}-V\|_{2}^{2}+\frac{1}{2}\|\nabla \tilde{V}\|_{2}^{2} .
$$

where $\alpha, \beta$ is penalty factor. $\tilde{V}$ is the optimized atmospheric veil. The first item on the right is called regular item, which ensures the smoothness of the processed image. The second term is called the total variation term, which ensures that the processed image can be as close as possible to the original image. To improve the computational speed of the above model, we use the neighborhood of $r \times r$ to approximate the gradient of the image. The model can be approximated as:

$$
E^{\prime}(\tilde{V})=\frac{\alpha}{2}(\tilde{V}-V)^{2}+\frac{\beta}{2}\left(\sum_{i=1}^{r^{2}-1}\left(\tilde{V}-\tilde{V}_{i}\right)\right)^{2} .
$$


where $\tilde{V}_{i}$ is the neighborhood pixel of $\tilde{V}$. Let the derivative of the above equation be 0 , so we can get:

$$
\tilde{V}^{j}=\frac{\alpha \cdot V+\beta \cdot \sum_{i=1}^{r^{2}-1} T_{i}^{j-1}}{\beta \cdot\left(\alpha+\left(r^{2}-1\right)\right)} .
$$

where $j$ is the number of iterations. The first term in the molecule is dependent on the input information and can be calculated only once during the iteration. The second term needs to be continually updated that is simply a neighborhood sum operation, and we can use the box filter[1] to further speed up the processing, so its complexity can reduce to $\mathrm{O}(1)$. Let the initial input of $\tilde{V}$ be $V$. We find that it can get better results when the parameter is $\alpha=3, \beta=3(j-1)$ and $r=\max \left(l_{h}, l_{w}\right) / 15$. where $l_{h}$ is the image height and $l_{w}$ is image width.

\section{Fault-tolerant mechanism based on the haze density feature}

The purpose of introducing the adjustment factor $\omega$ is to make the restored images after dehazing appear more real and natural. However, if it is set to a smaller fixed value, which will inevitably lead to texture area or sky area over-enhanced problem. On the contrary, it will lead to dehazing effect not obvious. To solve these problems, we optimize atmospheric veil by the flat distribution of the image.

Firstly, we use edge operator to detect the gradient of the degraded image in order to get binary image $G_{s}$ that reflects the flat characteristics of image. Then, the resulting binary image is further optimized by using the closed operation of mathematical morphology[7]. The operation is as follows:

$$
\Psi=G_{s} \oplus \Lambda_{\text {dilate }} \Theta \Lambda_{\text {erode }} .
$$

where $\Lambda_{\text {dilate }}$ is dilation structural operator and $\Lambda_{\text {erode }}$ is erosion structural operator. After obtaining the flat distribution map $\Psi$, we rewrite Equation (2) as:

$$
t(x)=1-\Psi^{\gamma} \cdot \frac{\tilde{V}(x)}{A} .
$$

where $\gamma$ is the gamma coefficient $(\gamma$ is 0.1 in this paper), which is to make an overall adjustment of the whole image according to the application requirements. Fig. 1 shows a process of the middle process, we can see that it can effectively distinguish the hazy image of the flat distribution map.

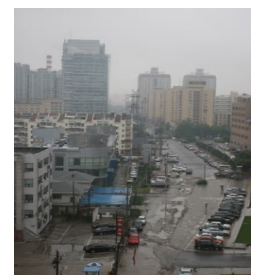

(a)

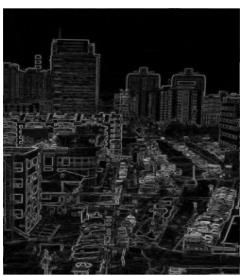

(b)

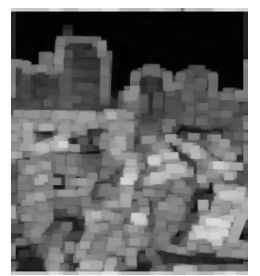

(c)

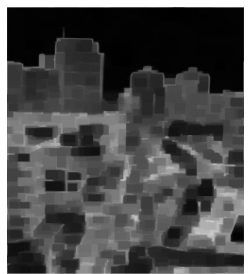

(d)

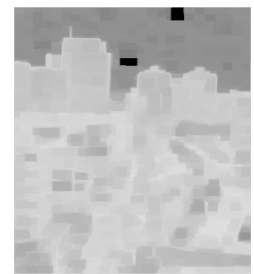

(e)

Fig. 1 middle processes to get flat distribution map: (a)hazy image; (b)gradient map; (c)dilation map; (d) erosion map; (e)flat distribution map.

\section{Image restoration}

We estimate atmospheric light A by dark channel prior theory. picking the top $0.1 \%$ brightest pixels in the dark channel. Among these pixels, the pixels with highest intensity in the input image I is selected as the atmospheric light[1].

We can get restored images by putting the estimated $\mathrm{A}$ and $\mathrm{t}(\mathrm{x})$ into the following equation:

$$
J(x)=\frac{I(x)-A}{\max \left(t(x), t_{0}\right)}+A
$$


when $\mathrm{t}(\mathrm{x})$ approaches 0 , the direct attenuation term tends to zero, which will make the restored image contain noise. Therefore, $\mathrm{t}_{0}$ is the lower limit for $\mathrm{t}(\mathrm{x})\left(\mathrm{t}_{0}\right.$ is 0.1 in this paper).

\section{Experimental Results}

In order to further verify the effectiveness of our algorithm, the recovery effect of our algorithm is compared with several mainstream dehazing algorithms shown in Fig.2. It can be seen that Tarel's algorithm[]ㅡ uses median filter to obtain the atmospheric veil that make the estimation of it inaccurate. Therefore, the recovery effect is poor and the color of the sky area is dim. Although Zhu's algorithm[6] has fast dehazing speed, its effect is not stable. When processing the hazy image, the degree of haze removal is not enough. He's algorithm[1] uses dark channel prior to restore image and its recovery effect is better in general. However, its computational complexity is very high, which make it difficult to apply to the actual scene. Ju's algorithm[4] has good dehazing effect and stability, but it is easy to regard large area of flat and bright object as dense haze that will cause over-enhancement phenomenon. Our method not only has obvious effect of dehazing, but also avoids the over-enhancement phenomenon when dealing with the sky area, so that the treated sky of restored image is very close to the real scene. At the same time, the dehazing speed of our algorithm also has certain advantages that its running time is basically the same as that of the Zhu's algorithm, which has the highest efficiency. As shown in Table 1.

Table 1. Comparison of running time of each algorithm

\begin{tabular}{cccccc}
\hline Image size & \multicolumn{5}{c}{ Algorithm[s] } \\
\cline { 2 - 6 } [pixel] & Tarel & zhu & He & Ju & our \\
\hline $291 \times 345$ & 3.294 & 1.149 & 33.035 & 6.001 & 1.366 \\
$384 \times 512$ & 9.995 & 1.154 & 59.219 & 9.400 & 1.467 \\
$459 \times 690$ & 24.461 & 1.486 & 94.862 & 14.884 & 1.822 \\
$497 \times 768$ & 32.043 & 1.544 & 120.429 & 18.958 & 2.096 \\
\hline
\end{tabular}
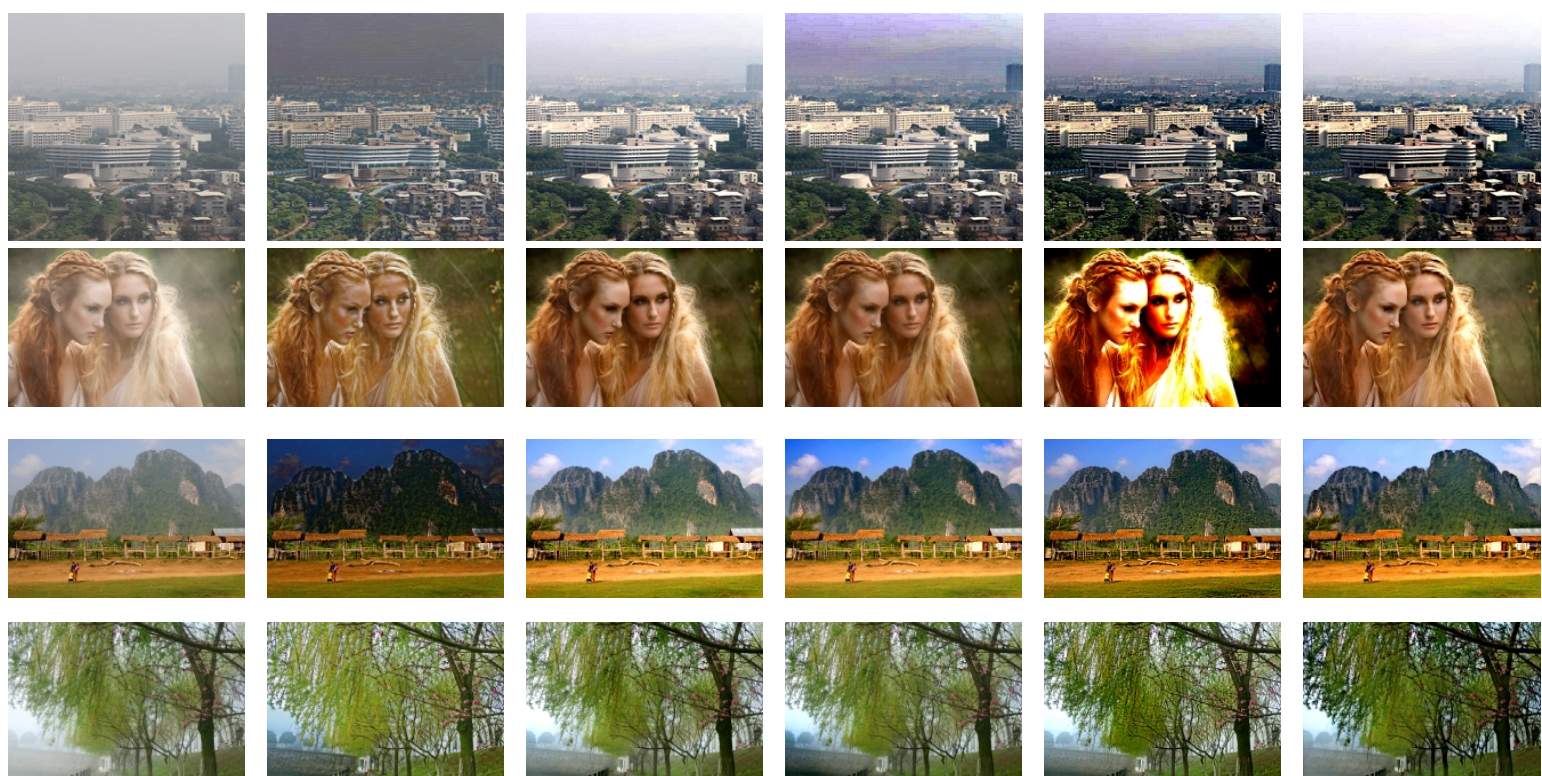

(d)

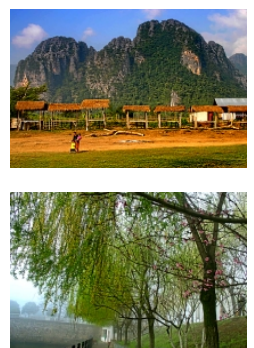

(e)

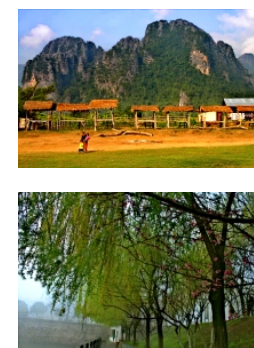

(f)

Fig.2 The result of dehazing techniques:(a)dehazed images; (b) Tarel; (c) Zhu; (d)He; (e)Ju; (f) our

In addition, we use the visible edge gradient ratio (corresponding to $r$ ) that is used to balance the contrast information of the restored image, the structural similarity (corresponding to $s$ ) that is used to measure the severity of the phenomenon of over-enhancement or Halo effect and the haze density index(corresponding to $\mathrm{d}$ ) as the objective evaluation index of restored image.Under normal circumstances, the greater of $\mathrm{r}$ or the smaller of $\mathrm{d}$ indicating the stronger dehazing ability, and the 
greater of s indicating that the effect of dehazing is more soft. The evaluation index for each group of examples in Fig. 2 is shown in Table 2. It can be seen from the data that Zhu's algorithm obtain larger $\mathrm{s}$, but its $\mathrm{r}$ is smaller and d is larger. Tarel's algorithm has lesser d, but its $\mathrm{s}$ is lesser. In contrast, He's algorithm, Ju's algorithm and our algorithm in this paper are more balanced in all respects. However, our algorithm has greater $r$ and $s$, lower $d$ than He's. Compared with Ju's algorithm, although $r$ is smaller than Ju's, but ours has greater s. The above analysis has the same conclusion as the subjective comparison.

Table 2. Image quality evaluation index

\begin{tabular}{ccccccccccccccccccc}
\hline Algorithm & \multicolumn{3}{c}{ Tarel } & \multicolumn{1}{c}{ Zhu } & \multicolumn{3}{c}{ He } & \multicolumn{3}{c}{ Ju } & \multicolumn{4}{c}{ our } \\
index & $\mathrm{r}$ & $\mathrm{S}$ & $\mathrm{d}$ & $\mathrm{r}$ & $\mathrm{S}$ & $\mathrm{d}$ & $\mathrm{r}$ & $\mathrm{S}$ & $\mathrm{d}$ & $\mathrm{r}$ & $\mathrm{S}$ & $\mathrm{d}$ & $\mathrm{r}$ & $\mathrm{S}$ & $\mathrm{d}$ \\
\hline $345 \times 291$ & 2.02 & 0.81 & 0.45 & 1.82 & 0.87 & 0.55 & 2.27 & 0.77 & 0.33 & 2.96 & 0.57 & 0.19 & 2.16 & 0.79 & 0.26 \\
$497 \times 768$ & 1.71 & 0.82 & 0.19 & 1.41 & 0.82 & 0.21 & 1.31 & 0.82 & 0.19 & 2.31 & 0.51 & 0.19 & 1.57 & 0.84 & 0.19 \\
$384 \times 512$ & 1.79 & 0.62 & 0.18 & 1.40 & 0.90 & 0.30 & 1.50 & 0.88 & 0.23 & 2.18 & 0.73 & 0.21 & 1.93 & 0.82 & 0.21 \\
$459 \times 690$ & 1.80 & 0.67 & 0.15 & 1.25 & 0.84 & 0.24 & 1.18 & 0.82 & 0.19 & 1.79 & 0.78 & 0.13 & 1.76 & 0.80 & 0.11 \\
\hline
\end{tabular}

\section{Conclusion and future work}

In this paper, by analysising Tarel's algorithm, we propose the following improvements for its defects: (1). The total variation model is used instead of the median filter of Tarel's method to improve the edge preserving property on the premise of improving original calculation efficiency. (2). Aiming at the failure of the bright area in the atmospheric veil, we propose a fault tolerance mechanism based on the haze density feature to improve the universality of the algorithm. The experimental results show that the dehazing effect of our algorithm is obvious that the restored image is clear and natural. It can effectively preserve the edge characteristics of the restored image and has low computational complexity. However, there are many parameters in the paper. In some special cases, the effect of dehazing is general. How to adjust the parameters adaptively is the point of our following study.

\section{Acknowledgement}

The work was partially supported by the National Natural Science Foundation of China [61571241], Industry-university-research Prospective joint project of Jiangsu Province [BY2014014], and Major projects of Jiangsu Province university natural science research [15KJA510002].

\section{References}

[1] He K M, Sun J, Tang X O. Single image haze removal using dark channel prior. In:Proceedings of the 2009 IEEE Conference on Computer Vision and Pattern Recognition.Miami, USA: IEEE, 2009. 1956-1963.

[2] He K, Jian S, Tang X. Guided image filtering.[J]. Pattern Analysis \& Machine Intelligence IEEE Transactions on, 2013, 35(6):1397-1409.

[3] Tarel J P, Hautiere N. Fast visibility restoration from a single color or gray level image[C]// Computer Vision,2009 IEEE 12th International Conference on. IEEE, 2009:2201-2208.

[4] Mingye Ju, Dengyin Zhang, Xuemei Wang. Single image dehazing via an improved atmospheric scattering model [J]. Visual Computer, 2016:1-13.

[5] Narasimhan S G, Nayar S K. Vision and the Atmosphere[J]. International Journal of Computer Vision, 2002,48(3):233-254.

[6] Zhu Q, Mai J, Shao L. A fast single image haze removal algorithm using color attenuation prior. 
IEEE Transactions on Image Processing, 2015, 24(11): 3522-3533.

[7] Ah Yin, Xing-yun. Operators to dilate and erode color impulse noise images based on the morphology. E -Business and E -Government (ICEE), 2011 International Conference on IEEE, 2011:1-3. 